\title{
LUM Smoother with Smooth Control for Noisy Image Sequences
}

\author{
Rastislav Lukáč \\ Department of Electronics and Multimedia Communications, Technical University of Košice, \\ Park Komenského 13, 04120 Košice, Slovak Republic \\ Email:lukacr@tuke.sk \\ Stanislav Marchevský \\ Department of Electronics and Multimedia Communications, Technical University of Košice, \\ Park Komenského 13, 04120 Košice, Slovak Republic \\ Email:marchs@tuke.sk
}

Received 7 December 2000 and in revised form 3 May 2001

\begin{abstract}
This paper focuses on adaptive structure of LUM (lower-upper-middle) smoothers for noisy image sequences. For the balance between noise suppression and signal-detail preservation, the LUM smoothers are widely used in smoothing applications. The amount of smoothing done by LUM smoothers is controlled by tuning parameter. However, the smoothing level is fixed for whole image. Thus, the excessive or insufficient smoothing can be performed. This problem is solved by a new method based on the adaptive controlled level of smoothing. A new method has excellent performance of the noise reduction in the environments corrupted by the impulse noise. In addition, minimal signal-detail and motion blurring can be observed. The performance of proposed method is evaluated through objective criteria and compared with traditional temporal, spatial, and spatiotemporal LUM smoothers.
\end{abstract}

Keywords and phrases: image sequence processing, impulse noise, LUM smoother, adaptive control.

\section{INTRODUCTION}

The processing of image sequences is widely used in medical imaging, computer and robot vision, video communications (e.g., phone video conferencing) and television transmission chain. However, the time-varying images or image sequences can be considered as spatiotemporal data [1], that is, a time sequence of two-dimensional (2D) images. The fact that the third dimension, that is, time is included increases computing complexity and time processing.

In practice, image signals interfere with impulse noise included by atmospheric noise, such as lightning spikes and spurious radio emission in radio communication, and relay switching noise in telephone channels. In addition to these natural non-Gaussian noise sources, there is a great variety of man-made sources such as electronic devices. It is evident that for sufficient restoration of degraded image signals some filtering techniques must be used. Therefore, few methods of the noise removing were developed. Concerning the filter input set, the noise removing methods have been divided into three classes (see Figure 1) such as temporal filtering, spatial (planar) filtering, and spatiotemporal filtering.

The class of temporal filters (see Figure 1a) is referred to temporal correlation of frames. One-dimensional filters remove noise without impairing the spatial resolution in stationary areas. In the case of large motion, the performance of temporal filters is insufficient $[1,2,3]$ and the temporal filtering must be connected with motion compensation [4] so as to filter objects along their motion trajectory. However, this way is very computationally complex, and because of spatial warping and scene changes, the motion compensation often does not work well.

2D spatial filters $[5,6,7]$ utilize the spatial correlation within the processed frame (see Figure 1b). These filters can be designed to give good filtering results without spatial blurring. However, the motion trajectory is not respected and motion blurring is introduced.

Three-dimensional filters (see Figure 1c) utilize the spatiotemporal correlation (i.e., temporal correlation of frames and spatial correlation without processed frame) present in image sequences. For that reason, the spatiotemporal filters can produce the best estimate in comparison with temporal and spatial filters. In addition, the spatiotemporal filtering $[1,2,3,8,9,10]$ can excellently remove the noise with the simultaneous signal blurring reduction (including spatial and motion blurring). 


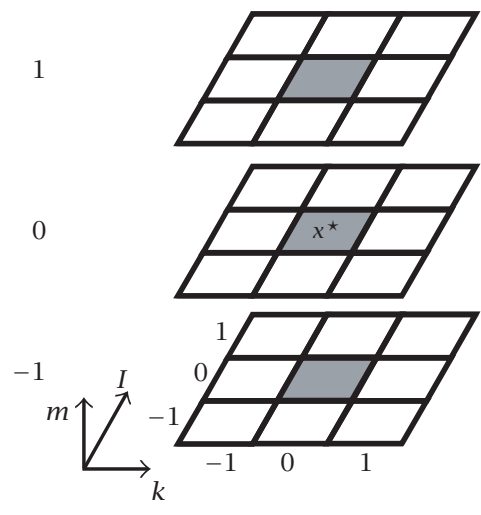

(a)

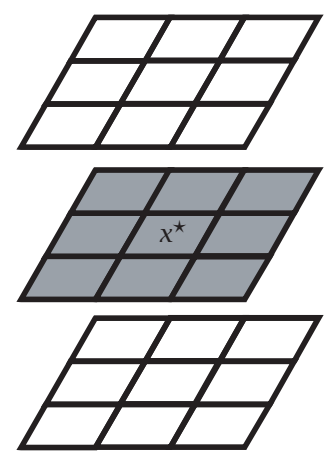

(b)

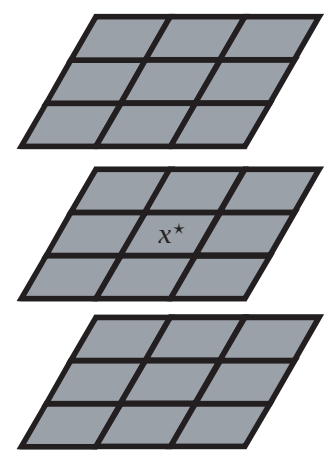

(c)

FIGURE 1: Filter windows for image sequence: (a) temporal, (b) $3 \times 3$ spatial, (c) cube spatiotemporal.

Many works related to spatiotemporal filtering show that median filters are not suitable for image sequence filtering due to their introducing of signal-details blurring. The blurring introduced by a filter may be more objectionable than the original noise. However, the median-related filters, especially, multistage median filters such as LUM (lower-uppermiddle) smoothers provide the best balance between impulse noise suppression and signal-detail preservation. Excellent smoothing and preserving characteristics can be obtained from filter structure by simply varying the tuning parameter for smoothing. However, smoothing level is fixed for a whole image, so the excessive or insufficient smoothing can be performed. Varying the level of smoothing dependently on local statistics must improve a restoration process, markedly.

The aim of this paper is to show that spatiotemporal filtering by LUM smoothers with adaptive smooth control can be effectively used in image sequence filtering and the superlative results can be obtained. In Section 2, image and noise models are described. Three objective criteria are defined, including criterion for the motion preservation. Section 3 focuses on the class of LUM smoothers, the results obtained by basic temporal, spatial, and spatiotemporal structures of LUM smoothers are presented. In Section 4, new method is proposed, the adaptive spatiotemporal LUM smoother is defined and described in detail. Algorithm of a smooth control is included. The properties of sequence filtering by LUM smoothers are concluded in Section 5.

\section{IMAGE AND NOISE MODELS}

As the test sequences, three image sequences of various statistical properties were used (see Figure 2). Each sequence consists of 30 frames with a resolution of $256 \times 256$ pixels with 8-bits/pixel gray-scale quantization. The complexity of image sequence is evaluated with regards to problem areas such as image details and edges. In addition, in case of image sequences the amount of motion is considered, too. The first sequence called Salesman (see Figures $2 a, 2 b$ ) includes a number of details and edges. The considerable motion of the man in a foreground increases complexity of this sequence. Sequence Susie (see Figures 2c, 2d) is the most elementary, there are many monotonous fields, the problem areas are hair. Accordingly, the minimal motion is observed. Small objects such as vegetation create background of sequence People (see Figures $2 \mathrm{e}, 2 \mathrm{f})$. In addition, there is a large complex motion caused by the motion of people and camera, simultaneously.

To illustrate the degree of damage, $10 \%$ impulse noise (simply I10) was used. The variable valued noise (see Figure 5a) replaces some of the image pixels by gray pixels, in case of 8 bit-quantized image by value between $0-255$. The mathematical formula for variable valued impulse noise is given by [3]

$$
x_{i j}= \begin{cases}o_{i j} & \text { with probability } 1-p, \\ z & \text { with probability } p,\end{cases}
$$

where $x_{i j}$ is noisy image signal, $o_{i j}$ describes original image signal and $i, j$ are indices of pixel location, $z$ is random value from $\langle 0,255\rangle$ and $p$ is the impulse probability.

To quantify the performance of the proposed method, a number of simulations were done. The results were compared regarding basis of subjective visual criterion and three objective criteria. Beside standard three-dimensional (3D) sequence objective criteria such as mean absolute error (MAE) and mean square error (MSE), the 3D statistical crosscorrelation $(\Delta R)$, [2], of the frames was verified. Thus, three measures were evaluated, that is, the detail preservation by MAE, the noise suppression by MSE, and the motion trajectory preservation by $\Delta R$.

For $n$th frame the mathematical formulas of standard MAE and MSE are given by

$$
\begin{aligned}
\operatorname{MAE}^{n} & =\frac{1}{N M} \sum_{i=1}^{N} \sum_{j=1}^{M}\left|o_{i, j}^{n}-x_{i, j}^{n}\right|, \\
\operatorname{MSE}^{n} & =\frac{1}{N M} \sum_{i=1}^{N} \sum_{j=1}^{M}\left(o_{i, j}^{n}-x_{i, j}^{n}\right)^{2},
\end{aligned}
$$




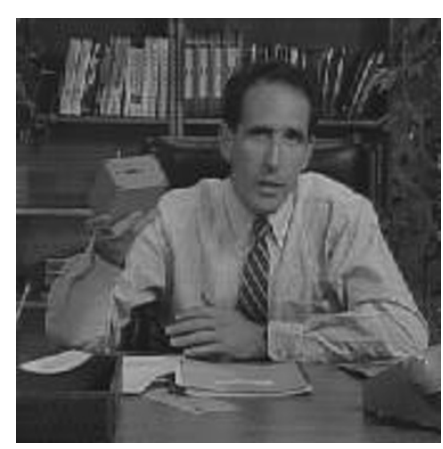

(a)

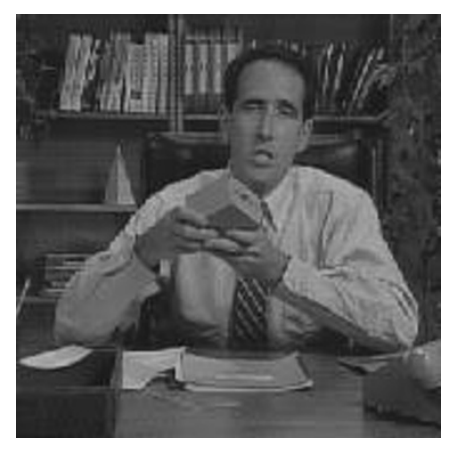

(b)

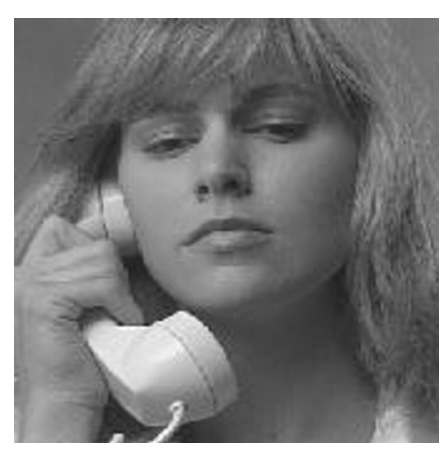

(c)

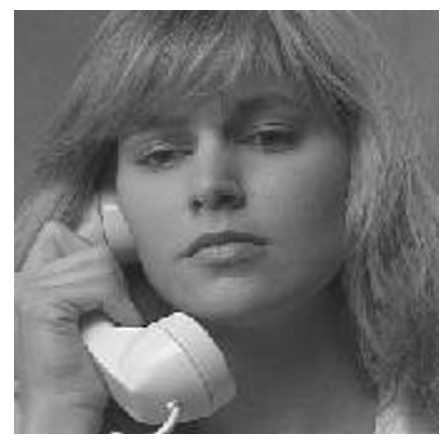

(d)

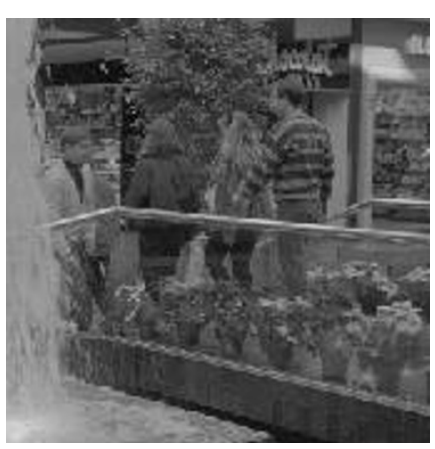

(e)

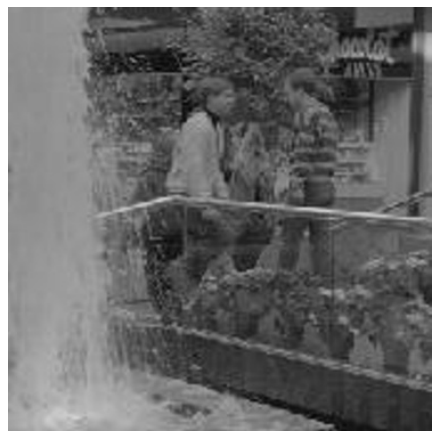

(f)

Figure 2: (a) Salesman-5th frame, (b) Salesman-25th frame, (c) Susie-5th frame, (d) Susie-25th frame, (e) People-5th frame, (f) People-25th frame.

where $\left\{o_{(i, j)}\right\}$ is the original image, $\left\{x_{(i, j)}\right\}$ is the filtered (distorted) image, $i, j$ are indices of image pixel position and $N, M$ are the image dimensions.

The 3D MAE and 3D MSE for whole sequence, [2], are defined as follows:

$$
\operatorname{MAE}=\frac{1}{F} \sum_{n=1}^{F} \operatorname{MAE}^{n}, \quad \operatorname{MSE}=\frac{1}{F} \sum_{n=1}^{F} \operatorname{MSE}^{n},
$$

where $F$ is the number of frames and $n$ is the frame index.

The motion trajectory preservation was quantified by a cross correlation $[2,3]$ given by

$$
\begin{aligned}
E^{n} & =\frac{1}{N M} \sum_{i=1}^{N} \sum_{j=1}^{M} x_{i, j}^{n}, \\
\sigma^{n} & =\sqrt{\frac{1}{N M} \sum_{i-1}^{N} \sum_{j-1}^{M}\left(x_{i, j}^{n}-E^{n}\right)^{2}}, \\
R^{n} & =\left|\frac{(1 / N M) \sum_{i=1}^{N} \sum_{j=1}^{M} x_{i, j}^{n} x_{i, j}^{n+1}-E^{n} E^{n+1}}{\sigma^{n} \sigma^{n+1}}\right|,
\end{aligned}
$$

where $E^{n}$ is the mean value of the $n$th frame of the sequence, $\sigma^{n}$ is the standard deviation of the $n$th frame and $R^{n}$ is the statistical cross correlation between the $n$th frame and the $(n+1)$ th frame. In like manner as above, for whole sequence the cross correlation is defined by

$$
R=\frac{1}{F} \sum_{n=1}^{F} R^{n}
$$

The best motion preservation is achieved by the smallest difference of cross correlation coefficients between the original noise-free sequence and the filtered sequence, as follows:

$$
\Delta R=\left|R^{\mathrm{o}}-R^{\mathrm{f}}\right|,
$$

where $R^{\mathrm{o}}$ and $R^{\mathrm{f}}$ are statistical cross-correlations of the original and filtered sequence, respectively.

Evaluation of results was achieved through the abovementioned criteria. Table 1 shows the degree of damage between original sequences and their distorted versions. Note that for objective criteria $N=M=256$. In the evaluation 15 pixels around the border were not used, therefore $N=M=226$. The pixels bypass around the border was on the ground of border effect [11]. For frame border, [2, 3], 3 frames around the border, were not considered. Thus, the number of considered frames was $F=24$. 
TABLE 1: Evaluation of noisy sequences.

\begin{tabular}{lccr}
\hline Criteria & 3D MAE & 3D MSE & $\Delta R$ \\
\hline Salesman & 7.287 & 825.1 & 0.396 \\
\hline Susie & 6.738 & 688.4 & 0.352 \\
\hline People & 7.069 & 772.8 & 0.741 \\
\hline
\end{tabular}

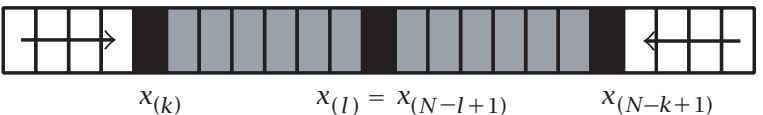

(a)

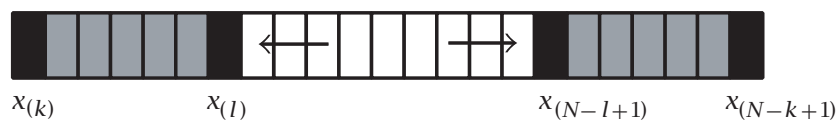

(b)

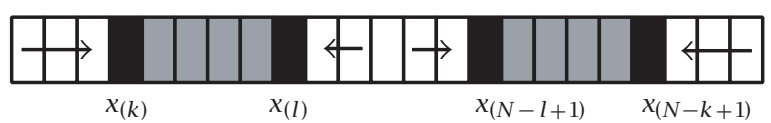

(c)

FIGURE 3: LUM filter as the (a) LUM smoother, (b) LUM sharpener, and (c) hybrid smoothing and sharpening filter.

\section{LUM SMOOTHER}

The LUM smoothers, a subclass of rank-order based LUM filters $[5,6,12]$ (see Figure 3 ) dispose by wide range of smoothing characteristics. The level of smoothing done by LUM smoother is given by tuning parameter for smoothing. Thus, the LUM smoothers can be designed to achieve the best balance between noise suppression and signal-details preservation.

Mathematically, the output of LUM smoother with parameter $k$ is given by

$$
y_{k}=\operatorname{med}\left\{x_{(k)}, x^{*}, x_{(N-k+1)}\right\},
$$

where "med" is the median operator that requires ordering of considered set and the choice of central sample from ordered set, $N$ is a window size, $x^{*}$ is a middle sample of input set, $x_{(k)}$ and $x_{(N-k+1)}$ are lower and upper order statistics defined as

$$
x_{(1)} \leq x_{(2)} \leq \cdots \leq x_{(N)} .
$$

Definition (7) is equivalent to center-weighted median (CWM), that is, given by the median over a modified set of observations that include multiple processed samples. Then, according to a multiple operator given by

$$
w_{i} \diamond x_{i}=\underbrace{x_{i}, x_{i}, \ldots, x_{i}}_{w_{i} \text { times }},
$$

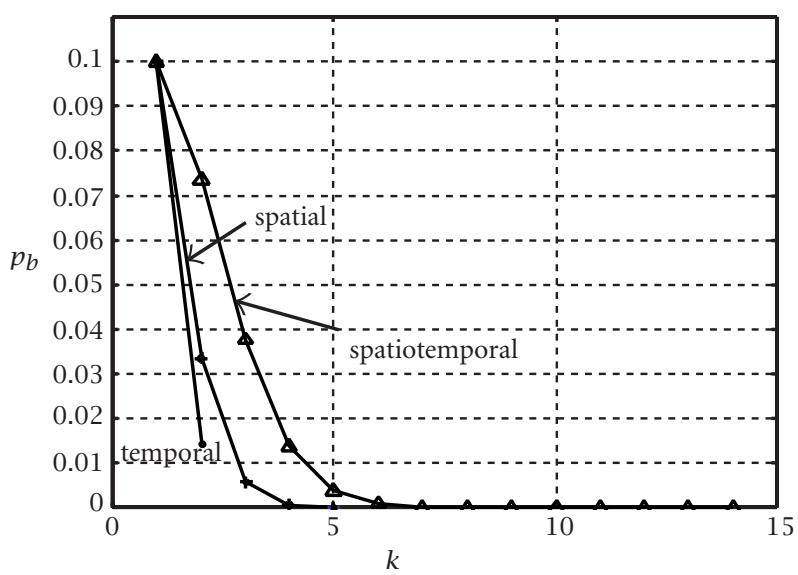

(a)

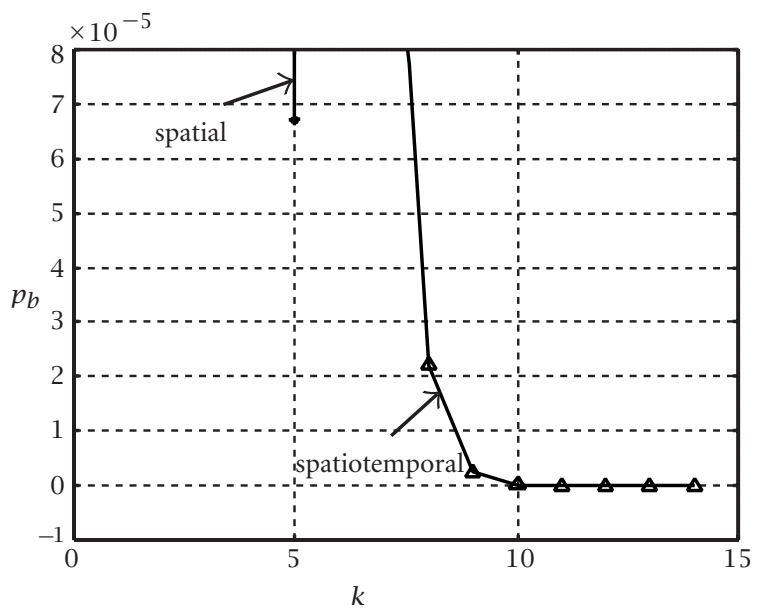

(b)

FIGURE 4: Breakdown probability of LUM smoother for temporal, spatial, and spatiotemporal windows. (a) full figure, (b) detail to $k=5,6, \ldots, 14$.

the output of LUM smoothers with parameter $k$ and a window size $N$ can be equivalently expressed as

$$
y_{k}=\operatorname{med}\left\{w_{1} \diamond x_{1}, w_{2} \diamond x_{2}, \ldots, w_{N} \diamond x_{N}\right\},
$$

where $x_{1}, x_{2}, \ldots, x_{N}$ are samples of input set and $w_{i}, i=$ $1,2, \ldots, N$, are filter weights defined by

$$
w_{i}= \begin{cases}N-2 k+2, & \text { for } i=\frac{N+1}{2}, \\ 1, & \text { otherwise. }\end{cases}
$$

However, implementation of the LUM smoother as shown in (7) requires fewer operations, [5], than that of (10), since fewer elements must be sorted.

Important is the fact that the output of LUM smoother is restricted to be a sample of input set and thus, it will never cause any undershoot and overshoot. The next required filter property is related to a blurring process. In many applications 


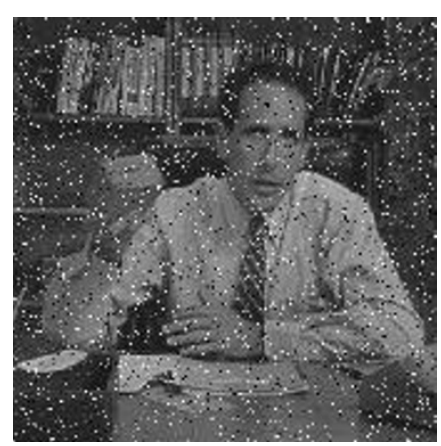

(a)

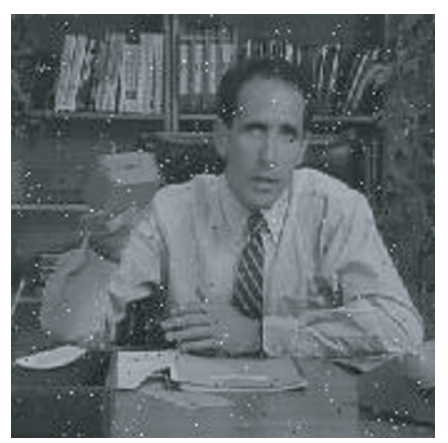

(b)

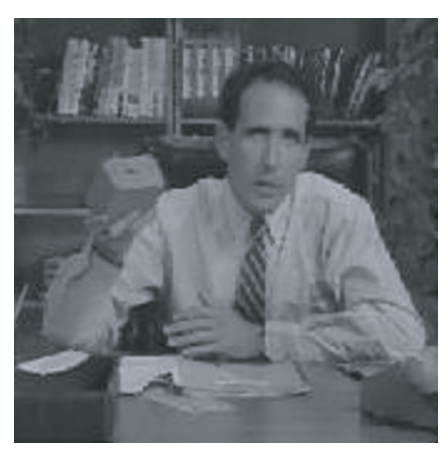

(c)

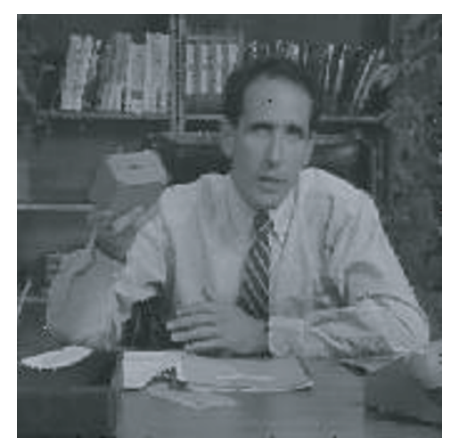

(d)

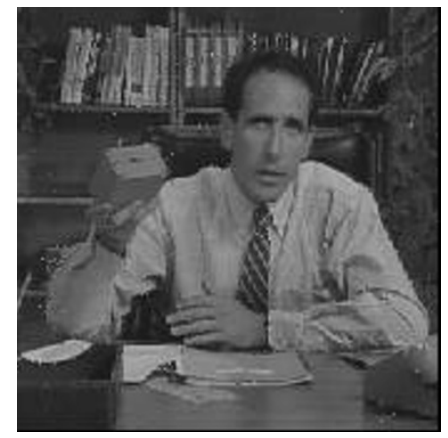

(e)

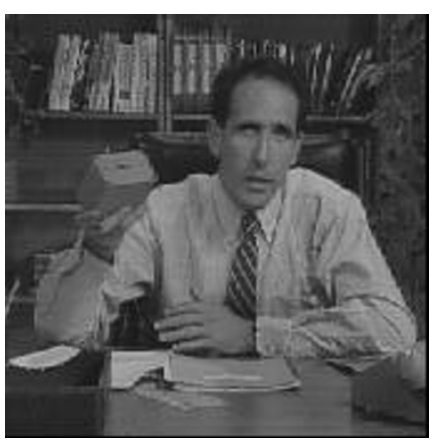

(f)

FIgURE 5: (a) I10 noise; I10 filtered by: (b) temporal median $(k=2)$, (c) $3 \times 3$ spatial median $(k=5)$, (d) $3 \times 3$ LUM smoother for $k=4$, (e) cube spatiotemporal LUM smoother for $k=6$, (f) new method-adaptive spatiotemporal LUM smoother.

some filters (typical example is a simple median) introduce too much smoothing that results in a blurring. The blurring introduced by a filter may be more objectionable than the original noise. In the case of LUM smoothers varying the filter parameter $k$ changes the level of the smoothing from no smoothing (i.e., identity filter for $k=1$, where central sample $x^{*}$ is passed to a filter output without any change) to the maximum amount of smoothing (i.e., median, where $k=(N+1) / 2)$. According to (7), smoothing function is created by simple comparing of processed sample $x^{*}$ with lower- and upper-order statistics. If $x^{*}$ lies in a range formed by these order statistics, it is not modified. If $x^{*}$ lies outside this range, it is replaced by a sample that lies closer to the median. When $k$ is chosen between maximum and minimum of possible parameter values, that is, $1<k<(N+1) / 2$ is valid, then filter exhibits the noise suppression property with the simultaneous signal-details preservation and a blurring process can be reduced.

From statistical properties, the very important impulse noise breakdown probability of LUM smoothers was derived, [5]. The breakdown probability is the probability of outputting an impulse given a certain probability $p$ of impulses appearing in the input (i.e., in the case of $10 \%$ noise, the probability $p=0.1$ ). Thus, the breakdown probability $p_{b}$ for the
LUM smoother is given by

$$
\begin{aligned}
p_{b}= & p \sum_{i=k-1}^{N-1}\left(\begin{array}{c}
N-1 \\
i
\end{array}\right)\left(\frac{p}{2}\right)^{i}\left(1-\frac{p}{2}\right)^{N-i-1} \\
& +(2-p) \sum_{i=N-k+1}^{N-1}\left(\begin{array}{c}
N-1 \\
i
\end{array}\right)\left(\frac{p}{2}\right)^{i}\left(1-\frac{p}{2}\right)^{N-i-1} .
\end{aligned}
$$

It is clear that the breakdown probability for LUM smoother (see Figure 4) is decreased with increased parameter $k$. When $p$ is small, a low breakdown probability can be obtained with relatively small $k$. By the low value of $k$ there can be achieved excellent signal-details preservation.

When a temporal window of three image points is considered, the LUM smoothers demonstrated the inefficient performance (see Table 4, Figure 5b). In this case, the tuning level $k$ was restricted to be from $\{1,2\}$. For $k=1$, the LUM smoother performs an identity operation. When the maximum amount of smoothing is done $(k=2)$, the LUM smoother is equivalent to a simple temporal median of three points, [2]. However, a temporal median produces a number of failure estimates, because if two or three samples of threeelement input set were corrupted, a filter estimate would be corrupted, too. 
TABLE 2: Evaluation of cube (spatiotemporal) LUM smoothers.

\begin{tabular}{|c|c|c|c|c|c|c|c|c|c|}
\hline \multirow{2}{*}{$k$} & \multicolumn{3}{|c|}{ Salesman I10 } & \multicolumn{3}{|c|}{ Susie I10 } & \multicolumn{3}{|c|}{ People I10 } \\
\hline & 3D MAE & 3D MSE & $\Delta R$ & 3D MAE & 3D MSE & $\Delta R$ & 3D MAE & 3D MSE & $\Delta R$ \\
\hline 1 & 7.287 & 825.1 & 0.396 & 6.738 & 688.4 & 0.337 & 7.069 & 772.8 & 0.352 \\
\hline 2 & 4.999 & 467.3 & 0.254 & 4.385 & 365.7 & 0.196 & 4.970 & 438.3 & 0.221 \\
\hline 3 & 3.070 & 215.5 & 0.126 & 2.503 & 153.9 & 0.085 & 3.303 & 210.1 & 0.105 \\
\hline 4 & 2.033 & 98.1 & 0.057 & 1.544 & 61.0 & 0.032 & 2.487 & 107.2 & 0.041 \\
\hline 5 & 1.621 & 54.3 & 0.029 & 1.190 & 28.7 & 0.012 & 2.223 & 68.1 & 0.010 \\
\hline 6 & 1.527 & 38.5 & 0.018 & 1.117 & 18.4 & 0.005 & 2.254 & 54.2 & 0.009 \\
\hline 7 & 1.595 & 33.0 & 0.012 & 1.168 & 15.0 & 0.002 & 2.452 & 49.5 & 0.022 \\
\hline 8 & 1.731 & 30.7 & 0.008 & 1.286 & 13.9 & 0.002 & 2.762 & 49.5 & 0.035 \\
\hline 9 & 1.948 & 31.0 & 0.005 & 1.454 & 13.9 & 0.004 & 3.177 & 53.0 & 0.047 \\
\hline 10 & 2.288 & 34.8 & 0.004 & 1.674 & 14.9 & 0.006 & 3.701 & 59.9 & 0.059 \\
\hline 11 & 2.603 & 36.9 & 0.005 & 1.944 & 16.5 & 0.007 & 4.315 & 69.2 & 0.069 \\
\hline 12 & 2.978 & 40.5 & 0.008 & 2.282 & 19.2 & 0.009 & 5.038 & 81.6 & 0.078 \\
\hline 13 & 3.521 & 48.4 & 0.010 & 2.712 & 23.2 & 0.010 & 5.912 & 98.5 & 0.084 \\
\hline 14 & 4.237 & 59.6 & 0.011 & 3.265 & 29.2 & 0.011 & 6.982 & 120.8 & 0.088 \\
\hline
\end{tabular}

TABLE 3: Evaluation of spatial $3 \times 3$ LUM smoothers.

\begin{tabular}{|c|c|c|c|c|c|c|c|c|c|}
\hline \multirow{2}{*}{$k$} & \multicolumn{3}{|c|}{ Salesman I10 } & \multicolumn{3}{|c|}{ Susie I10 } & \multicolumn{3}{|c|}{ People I10 } \\
\hline & 3D MAE & 3D MSE & $\Delta R$ & 3D MAE & 3D MSE & $\Delta R$ & 3D MAE & 3D MSE & $\Delta R$ \\
\hline 1 & 7.287 & 825.1 & 0.396 & 6.738 & 688.4 & 0.337 & 7.069 & 772.8 & 0.352 \\
\hline 2 & 3.048 & 219.2 & 0.135 & 2.500 & 160.0 & 0.094 & 3.117 & 204.7 & 0.110 \\
\hline 3 & 2.029 & 61.3 & 0.033 & 1.521 & 34.0 & 0.013 & 2.371 & 61.2 & 0.009 \\
\hline 4 & 2.648 & 46.1 & 0.009 & 1.956 & 21.2 & 0.003 & 3.258 & 50.7 & 0.022 \\
\hline 5 & 4.097 & 64.3 & 0.003 & 3.097 & 30.9 & 0.006 & 5.161 & 76.9 & 0.038 \\
\hline
\end{tabular}

Spatial LUM smoothers showed better effect of the impulse noise suppression (see Table 3). The characteristic feature of LUM smoothers with control parameter $k=3,4$ is the best balance between noise suppression and detail preservation. In some situations, it was observed that these filters can produce artifacts in the form of motion blurring.

For that reason, most appropriate filters for image sequences have a spatiotemporal base combining properties of temporal and spatial filters. When the most frequently used cube window over three frames is considered, it increases a number of possible parameter values for spatiotemporal LUM smoothers. Note that for cube window $(N=27)$ a number of possible LUM smoothers is $(N+1) / 2=14$. The mentioned fact can be a problem in getting the best results. Arce [8] shows that LUM smoothers with tuning level $k=6$ (see Figure 5e) are most appropriate for sequence filtering. These results were confirmed (see Table 2) and we add that the best results for our test sequences were achieved for parameter values $k=5,6,7$. In addition, the LUM smoothers with parameter values $k=5,6,7$ cause minimal spatial and motion blurring.

\section{PROPOSED METHOD}

Standard LUM smoothers use fixed smoothing level for a whole image. Thus, excessive or insufficient smoothing can be performed. The excessive smoothing can be observed as spatial or motion blurring. The insufficient amount of smooth- ing is characterized by some impulse presence. These drawbacks of standard LUM smoothers are removed by a new method called the cube spatiotemporal LUM smoother with adaptive control of smoothing.

The principle of the proposed method is shown in Figure 6. In the first step, outputs of standard LUM smoothers for all possible parameter values $k=1,2, \ldots,(N+1) / 2$ are computed. Specifically, in the case of spatiotemporal cube window $(N=27)$ are achieved 14 outputs. Next, absolute differences $d_{k}$, for $k=1,2, \ldots,(N+1) / 2$, are given by a simple comparison between central sample $x^{*}$ and standard LUM smoother output. Mathematically, this comparison can be expressed as

$$
d_{k}=\left|x^{*}-y_{k}\right|, \quad \text { for } k=1,2, \ldots, \frac{N+1}{2} .
$$

In the comparator array, the set of absolute differences $d_{1}, d_{2}, \ldots, d_{(N+1) / 2}$ is compared with the set of thresholds $\mathrm{Tol}_{1}, \mathrm{Tol}_{2}, \ldots, \mathrm{Tol}_{(N+1) / 2}$ for each possible $k$, separately. The condition for comparators $c_{k}$ is defined by

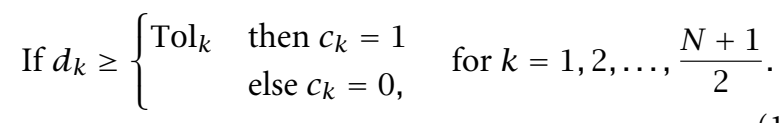

Finally, the output of the proposed method is given by

$$
y=y_{k_{\mathrm{opt}}}
$$


TABLE 4: Evaluation of temporal LUM smoothers (3 frames were considered).

\begin{tabular}{|c|c|c|c|c|c|c|c|c|c|}
\hline \multirow{2}{*}{$k$} & \multicolumn{3}{|c|}{ Salesman I10 } & \multicolumn{3}{|c|}{ Susie I10 } & \multicolumn{3}{|c|}{ People I10 } \\
\hline & 3D MAE & 3D MSE & $\Delta R$ & 3D MAE & 3D MSE & $\Delta R$ & 3D MAE & 3D MSE & $\Delta R$ \\
\hline 1 & 7.287 & 825.1 & 0.396 & 6.738 & 688.4 & 0.337 & 7.069 & 772.8 & 0.352 \\
\hline 2 & 3.086 & 109.1 & 0.029 & 2.457 & 89.2 & 0.022 & 5.882 & 194.4 & 0.035 \\
\hline
\end{tabular}

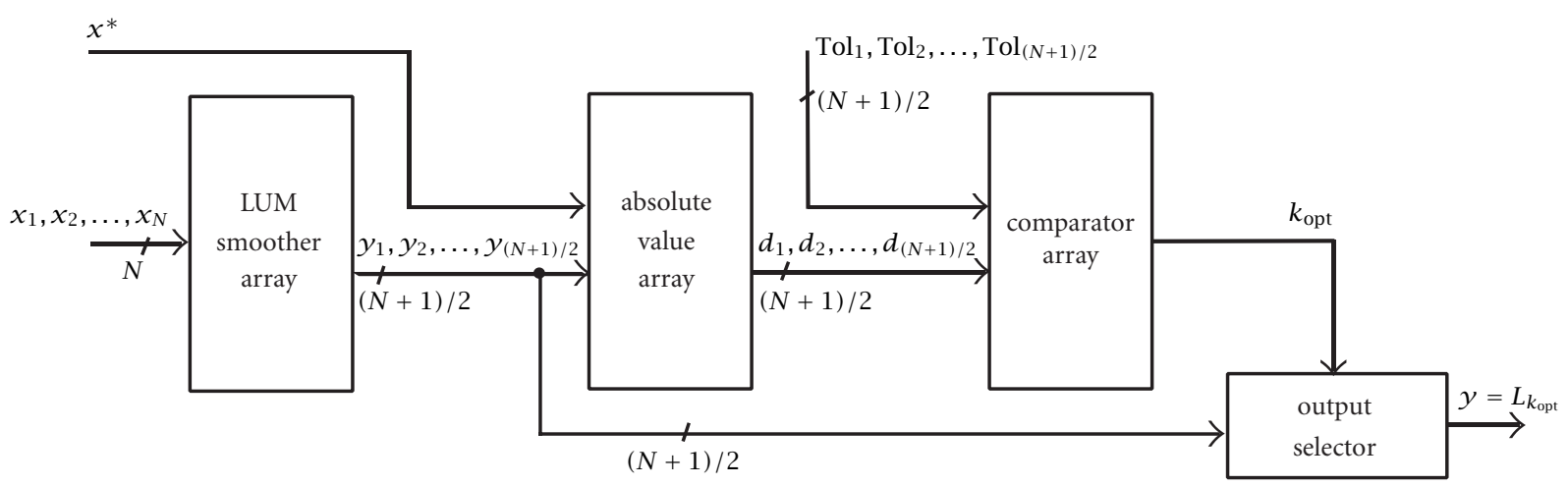

FIgURE 6: The structure of adaptive LUM smoother.

where

$$
k_{\mathrm{opt}}=\sum_{k=1}^{(N+1) / 2} c_{k}
$$

is constrained to be from $1 \leq k_{\text {opt }} \leq(N+1) / 2$. In (14) and (15), $c_{1}, c_{2}, \ldots, c_{(N+1) / 2}$ characterise the outputs of comparator array. Note that $c_{1}=1$ and $\mathrm{Tol}_{1}=0$, since threshold Tol corresponds to identity filter.

Thus, in every location of running window the output of adaptive LUM smoother is equal to smoothing level $k_{\text {opt }}$ corresponding to the sum of comparator outputs. The amount of smoothing done by adaptive LUM smoother is equal to the most appropriate value determined by output of traditional LUM smoother.

The only problem is to find the set of optimal thresholds so that the proposed method would suppress the noise with preserving image details simultaneously. There are $((N+1) / 2-1)$ ! possibilities that for $N=27$ (a cube window) is equal to 6227020800 . For that reason, it is not easy to find out the set of optimal thresholds. A genetic algorithm, [13], is an appropriate optimization method to find out an optimal solution.

Genetic algorithm provides the means to solve difficult optimization problems. It starts with a population of randomly generated solutions of the problem. In the case of the proposed method, the set of thresholds $\mathrm{Tol}_{1}, \mathrm{Tol}_{2}, \ldots$, $\mathrm{Tol}_{(N+1) / 2}$ was randomly generated. The next population is achieved by applying crossover and mutation operators. A crossover operator generates from two individuals of a current population an offspring. A random information is introduced to an offspring by a mutation operator. In this way, the next population is created. However, by a selection operator the size of the population is reduced typically to the size of the initial population. In this paper, the population consists of 120 chromosomes. Probabilities of crossover and mutation were 0.9 and 0.05 , respectively. The suboptimal set of thresholds $\mathrm{Tol}_{1}, \mathrm{Tol}_{2}, \ldots, \mathrm{Tol}_{14}$, written as $\mathrm{Tol}_{\text {opt }}=\{0,4,5,7,9,12,15,16,22,23,38,43,48,52\}$ was achieved after 3500 iterations approximately.

Now, we provide an example of algorithm performance. The example focuses on the input set with some noisy samples, including the noisy central sample. With this scheme we want to show the noise suppression property of the method. On the other hand, by considering the original input set we show the presentation of the signal-details preservation and the estimation with minimal error, as well.

Example 1. Let Figure 7a show an input original set determined by a cube filter window for following filter processing. Corrupted input set is provided in Figure $7 \mathrm{~b}$, where 6 outliers include the central sample are marked by a gray color. Since the corrupted set includes 27 samples, after applying (7) and (8) we obtain 14 outputs of the standard LUM smoothers ordered from IF to median, namely

$$
\begin{aligned}
& \left\{y_{1}, y_{2}, \ldots, y_{14}\right\} \\
& \quad=\{21,21,49,49,49,50,50,51,51,52,53,53,53,54\} .
\end{aligned}
$$

The problem is to determine concrete value, that is, $y_{k}$ that would estimate an original central sample with minimal error. Now, excellent noise suppression and signal detail preservation properties of proposed methods are required. According to (13) and the noisy central sample $x^{*}=21$ (see Figure 7b), 
TABLE 5: Evaluation of proposed method-adaptive spatiotemporal LUM smoother of cube window.

\begin{tabular}{|c|c|c|c|c|c|c|c|c|}
\hline \multicolumn{3}{|c|}{ Salesman I10 } & \multicolumn{3}{|c|}{ Susie I10 } & \multicolumn{3}{|c|}{ People I10 } \\
\hline 3D MAE & 3D MSE & $\Delta R$ & 3D MAE & 3D MSE & $\Delta R$ & 3D MAE & 3D MSE & $\Delta R$ \\
\hline 0.733 & 16.1 & 0.006 & 0.518 & 8.0 & 0.002 & 1.296 & 32.9 & 0.017 \\
\hline
\end{tabular}



(a)

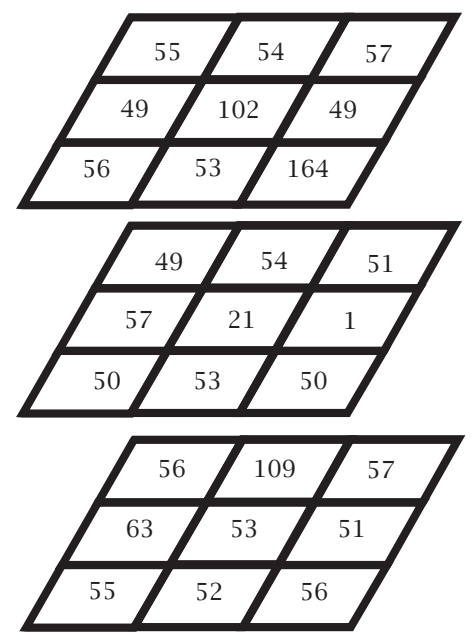

(b)

FIGURE 7: Example of the input set configuration: (a) original (noise free) input set, (b) noisy input set with 6 outliers.

we get the set of differences

$$
\begin{aligned}
& \left\{d_{1}, d_{2}, \ldots, d_{14}\right\} \\
& \quad=\{0,0,28,28,28,29,29,30,30,31,32,32,32,33\} .
\end{aligned}
$$

According to (14), if the set of differences $d_{k}$ for $k=$ $1,2, \ldots, 14$ is compared with the set of suboptimal thresholds Tol $_{\text {opt }}=\{0,4,5,7,9,12,15,16,22,23,38,43,48,52\}$ we can see that only differences $d_{k}$ for $k=1,3,4,5,6,7,8,9,10$ satisfy (14), then given by (16) $k_{\mathrm{opt}}=9$ and the proposed method produces an output that satisfies (15).

Finally, the output of the proposed method (15) is determined as $y_{k_{\mathrm{opt}}}=y_{9}=51$, which is identical with original central sample according to Figure 7a.

Results (see Table 5 and Figure 5f) indicate that the proposed method with sub-optimal set $\mathrm{Tol}_{\text {opt }}$ provides excellent noise suppression with simultaneous signal-detail preservation. The achieved results are characterized by a very small difference in comparison with original sequences in the sense of used objective criteria. In addition, the proposed method is robust and it does not depend on the mean intensity of used signals that is evident from following Tables 6 and 7.

Mean intensities of used test sequences and their noisy equivalents are provided in Table 6 . These sequences are taken approximately from identical range of mean intensities. To examine the performance of the proposed method in sequences with considerable different mean intensities, we
TABLE 6: Mean intensity of used sequences.

\begin{tabular}{lccc}
\hline Sequence & Salesman & Susie & People \\
\hline original & 82.107 & 110.288 & 86.645 \\
\hline noisy & 86.324 & 111.808 & 90.461 \\
\hline
\end{tabular}

use three modifications of sequence Salesman (see Table 7 and Figure 8), where we perform in the first step an intensity shift whereas in the second step these modified original sequences with different mean intensities were corrupted by $10 \%$ impulse noise. After this the proposed adaptive LUM smoother with identical set of thresholds $\left(\right.$ Tol $_{\text {opt }}=$ $\{0,4,5,7,9,12,15,16,22,23,38,43,48,52\})$ was applied. If the mean intensity of image sequence is near to light or dark intensities, then the degree of the noise corruption is higher, however, it cannot influence the performance of the proposed method (see Table 7). These results confirm that the proposed method is independent of the intensity range. In addition, in the case of realizing the intensity shift of nonoriginal but noisy sequences, it will exercise translation invariant operation. It means that the identical shift of input samples will result in the same shift on a filter output.

Although, this paper is focused on the filtering of noisy sequences and thus, the proposed method was optimized for image sequences, that is, spatiotemporal data, the primary purpose of all designed filter algorithms is related with the processing of still images, that is, 2D images. Similarly, the proposed method represents no exception. 


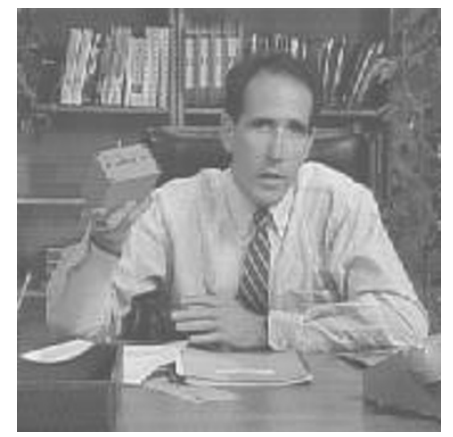

(a)

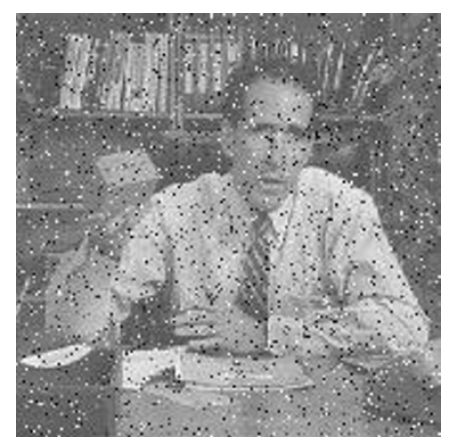

(b)

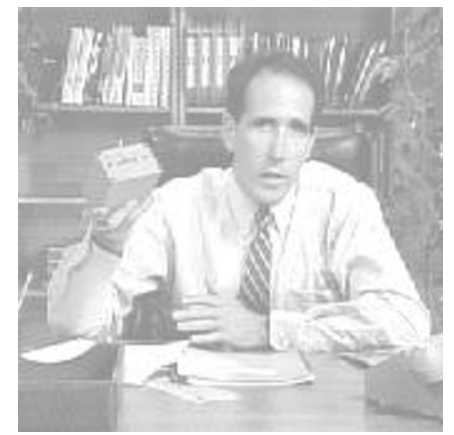

(c)

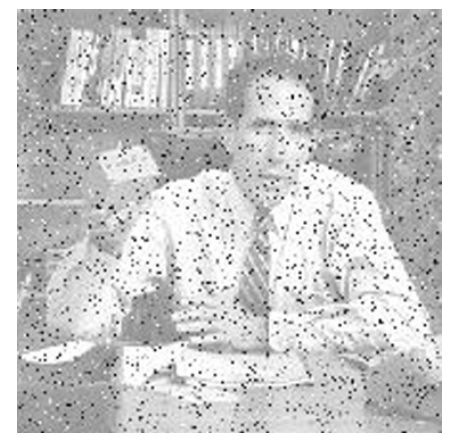

(d)

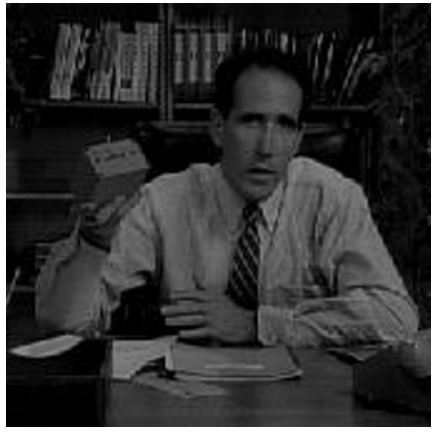

(e)

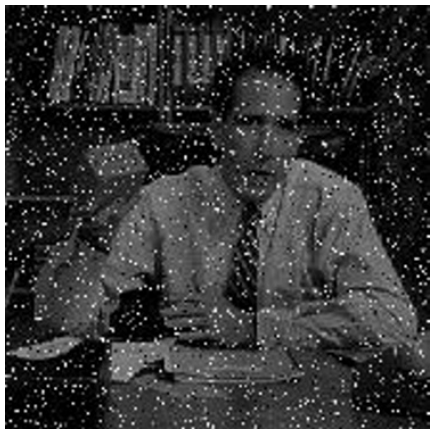

(f)

FIGURE 8: 5th frame of sequence Salesman (a) mean intensity 142.107, (b) noisy equivalent, (c) mean intensity 200.733, (d) noisy equivalent, (e) mean intensity 42.709, (f) noisy equivalent.

TABLE 7: Evaluation of proposed method on sequence Salesman with modified mean intensity $E$.

\begin{tabular}{cccccc}
\hline$\Delta E$ & Sequence & $E$ & 3D MAE & 3D MSE & $\Delta R$ \\
\hline \multirow{2}{*}{0} & original & 82.107 & - & - & - \\
\multirow{2}{*}{ Figure 2a) } & noisy & 86.324 & 7.287 & 825.1 & 0.396 \\
& filtered & - & 0.733 & 16.1 & 0.006 \\
\hline \multirow{2}{*}{+60} & original & 142.107 & - & - & - \\
$($ Figures 8a, b) & noisy & 140.722 & 6.785 & 698.1 & 0.364 \\
& filtered & - & 0.706 & 16.0 & 0.009 \\
\hline \multirow{2}{*}{+120} & original & 200.733 & - & - & - \\
$($ Figures 8c, d) & noisy & 195.420 & 8.800 & 1222.2 & 0.523 \\
& filtered & - & 0.642 & 14.4 & 0.010 \\
\hline \multirow{2}{*}{-40} & original & 42.709 & - & - & - \\
\multirow{2}{*}{ Figures 8e, 8f) } & noisy & 50.772 & 8.852 & 1228.1 & 0.506 \\
& filtered & - & 0.712 & 16.6 & 0.010 \\
\hline
\end{tabular}

Particular analysis with a similar improvment (approximately 40-70\% in comparison with standard median and 35$50 \%$ opposite standard LUM smoothers) such as in the case of image sequences was provided in [12, 14]. In addition, in [14] smoothing levels of standard LUM smoothers are controlled according to standard deviation. Full analysis of the adaptive LUM smoothers is presented in [15], where the method was extended to the next dimension of image information, that is, with the utilization of the color information resulting from vector processing $[16,17]$. Just [15] brings further results according to the dimension of processing and the range of the noise corruption. These results exclude the use of proposed adaptive LUM smoothers in the environments corrupted by the Gaussian noise. However, in the case of mixed noise that is combination of Gaussian noise and impulsive noise, the use of the proposed methods can remove the outliers and in the next filter stage the Gaussian noise can be suppressed by some L filter [18] or LI filter [19]. The mentioned approach can result in excellent image restoration.

If comparing the complexity of the proposed method for 2D images and image sequences, it is evident that a cube structure of the method for image sequences covers all important features including spatial and temporal changes of image signals. However, in some applications processing of 27 samples and following consideration of 14 outputs of LUM smoothers can consume more time than it is expedient. For that reason, we provide modification of the proposed method for a cube spatiotemporal window, where only six outputs of standard LUM smoothers are calculated. For comparison, in the case of standard $3 \times 3$ window for $2 \mathrm{D}$ still images, it is required five outputs. Thus, spatial 
and temporal features of sequences remain to be considered with the markedly decreasing computational complexity.

In the modified structure, we considered IF and median, that is, both extreme outputs of LUM smoothers. The IF is important in noise free regions, since the use of this filter produce no error. On the other hand, if some samples are corrupted, then only LUM smoothers with large value of $k$ can remove outliers, and thus median is equally important. Additional four filters are chosen from inside the set bounded by both extreme filters. Thus, we divide this set linearly and only LUM smoothers for $k=3,6,9,12$ are used. The new set of six LUM smoothers can be expressed as

$$
\left\{y_{1}^{\prime}, y_{2}^{\prime}, \ldots, y_{6}^{\prime}\right\}=\left\{y_{1}, y_{3}, y_{6}, y_{9}, y_{12}, y_{14}\right\}
$$

and their corresponding thresholds are simply written as

$$
\begin{aligned}
\left\{\operatorname{Tol}_{1}^{\prime}, \operatorname{Tol}_{2}^{\prime}, \ldots, \mathrm{Tol}_{6}^{\prime}\right\} & =\left\{\mathrm{Tol}_{1}, \mathrm{Tol}_{3}, \mathrm{Tol}_{6}, \mathrm{Tol}_{9}, \mathrm{Tol}_{12}, \mathrm{Tol}_{14}\right\} \\
& =\{0,5,12,22,43,52\} .
\end{aligned}
$$

From above it is evident that (13) must be modified to the expression given by

$$
d_{l}^{\prime}=\left|x^{*}-y_{l}^{\prime}\right|
$$

and the output of modified method is determined by

$$
y=y_{l_{\mathrm{opt}}}^{\prime} .
$$

Note that $l_{\mathrm{opt}}$ is forced to be from $1 \leq l_{\mathrm{opt}} \leq 6$, since

$$
l_{\mathrm{opt}}=\sum_{l=1}^{6} c_{l}^{\prime}
$$

where parameters $c_{l}^{\prime}$ for $l=1,2, \ldots, 6$ are given by

$$
\text { If } d_{l}^{\prime} \geq\left\{\begin{array}{rr}
\operatorname{Tol}_{l} & \text { then } c_{l}^{\prime}=1 \\
& \text { else } c_{l}^{\prime}=0,
\end{array} \text { for } l=1,2, \ldots, 6\right.
$$

Note that six thresholds for the modified method are not optimized for this case, but they were chosen and renumbered from the original optimized set of thresholds, only. This simplification is faster than the original adaptive LUM smoother for cube window, since from 2 to 3 times fewer operations must be performed, however, without much reduction in the overall quality (see Table 8 ).

Example 2. Consider identical original and noisy input set (see Figure 7) as Example 1, where central sample $x^{*}=21$. In the first step, we need to compute only 6 outputs of LUM smoothers

$$
\left\{y_{1}^{\prime}, y_{2}^{\prime}, \ldots, y_{6}^{\prime}\right\}=\left\{y_{1}, y_{3}, y_{6}, y_{9}, y_{12}, y_{14}\right\},
$$

namely

$$
\left\{y_{1}^{\prime}, y_{2}^{\prime}, \ldots, y_{6}^{\prime}\right\}=\{21,49,50,51,53,54\} .
$$

According to (21), it is necessary to obtain the set of differences

$$
\left\{d_{1}^{\prime}, d_{2}^{\prime}, \ldots, d_{6}^{\prime}\right\}=\{0,28,29,30,32,33\},
$$

that is, compared with the modified set of thresholds

$$
\left\{\operatorname{Tol}_{1}^{\prime}, \operatorname{Tol}_{2}^{\prime}, \ldots, \operatorname{Tol}_{6}^{\prime}\right\}=\{0,5,12,22,43,52\}
$$

to determine parameters $c_{l}^{\prime}(24)$. Then, a number of satisfied conditions $d_{l}^{\prime} \geq \mathrm{Tol}_{l}$, that is equal to 4 (for $l=1,2,3,4$ ) determines that $l_{\mathrm{opt}}=4$ (23). Finally, the output of modified method (22) is expressed as $y=y_{4}^{\prime}=51$, which means that the noisy central sample was replaced by a sample equal to the original value. This result confirms that the modified method can suppress the impulse noise nearly identically with the original adaptive concept.

The performance of the modified adaptive method is evaluated in Table 8. In comparison with results (see Table 5), achieved by original adaptive concept (15), it is evident that in the case of image sequences with small amount of motion such as Salesman and Susie, the modified methods achieve slightly worse results than the original adaptive concept. However, in the case of sequence with shining motion (e.g., People), the modified method can improve motion preservation with the simultaneous excellent MAE and MSE.

\section{CONCLUSION}

In this paper, a new method of LUM smoothing for image sequences has been developed, presented and compared with the traditional temporal, spatial and spatiotemporal LUM smoothers. The traditional LUM smoothers perform the noise reduction with the fixed level of smoothing for the whole image. Thus, excessive or insufficient smoothing can be performed. In the proposed method, such constraints do not exist. This makes the proposed method more preferable in the environments corrupted by impulse noise, where the excellent results were obtained. The proposed adaptive LUM smoother is simple and successful in smoothing applications. Excellent results are achieved by the adaptive controlled level of smoothing done by LUM smoother.

However, in some applications the computational complexity of the methods could result in time delay. For these cases we provide a simplified scheme of the proposed method, where only 6 from 14 possible standard LUM smoothers are computed and used to determine a filter output. The proposed simplified method achieves comparable results with the original adaptive method, however from 2 to 3 times fewer operations must be performed.

Both proposed methods can be used in practical applications, where impulse noise can corrupt useful image information. Achieved robust set of optimal thresholds can be fully applicable for estimating the samples of noisy image sequences with various statistical properties and the amount of signal complexity. 
TABLE 8: Evaluation of proposed simplified adaptive spatiotemporal LUM smoother with a cube window.

\begin{tabular}{|c|c|c|c|c|c|c|c|c|}
\hline \multicolumn{3}{|c|}{ Salesman I10 } & \multicolumn{3}{|c|}{ Susie I10 } & \multicolumn{3}{|c|}{ People I10 } \\
\hline 3D MAE & 3D MSE & $\Delta R$ & 3D MAE & 3D MSE & $\Delta R$ & 3D MAE & 3D MSE & $\Delta R$ \\
\hline 0.749 & 16.8 & 0.007 & 0.544 & 8.6 & 0.002 & 1.266 & 32.9 & 0.014 \\
\hline
\end{tabular}

\section{ACKNOWLEDGEMENT}

This work was supported by the Grant Agency of the Ministry of Education and Academy of Science of the Slovak Republic VEGA under Grant No. 1/5241/98.

\section{REFERENCES}

[1] T. Viero and Y. Neuvo, "3-D median structures for image sequence filtering and coding," in Motion Analysis and Image Sequence Processing, pp. 411-445. Kluwer Academic Publishers, 1993.

[2] R. Lukáč, C. Stupák, S. Marchevský, and L. Maceková, "Order statistic filters in dynamic image sequences," Radioengineering, vol. 9, no. 3, pp. 8-14, 2000.

[3] R. Lukáč, C. Stupák, and S. Marchevský, "Neural smoothers for noised dynamic image sequences," J. of Electr. Engrg., vol. 51, no. 11-12, pp. 312-317, 2000.

[4] M. K. Ozkan, M. I. Sezan, and A. M. Tekalp, "Adaptive motion-compensated filtering of noisy image sequences," IEEE Trans. on Circuits and Systems for Video Technology, vol. 3, no. 4, pp. 277-290, 1993.

[5] R. C. Hardie and C. G. Boncelet, "LUM filters: a class of rankorder-based filters for smoothing and sharpening," IEEE Trans. on Signal Processing, vol. 41, no. 3, pp. 1061-1076, 1993.

[6] R. C. Hardie and C. G. Boncelet, "Gradient-based edge detection using nonlinear edge enhancing prefilters," IEEE Trans. on Image Processing, vol. 4, no. 11, pp. 1572-1578, 1995.

[7] S. Marchevský and J. Chmúrny, "Two-dimensional digital filter with small generation kernels," J. of Electr. Engrg., vol. 35, pp. $872-875,1984$.

[8] G. R. Arce, "Multistage order statistic filters for image sequence processing," IEEE Trans. on Signal Processing, vol. 39, no. 5, pp. 1146-1163, 1991.

[9] R. Lukáč, C. Stupák, and S. Marchevský, "Filtering the grayscale image sequences by neural network," in 15th Biennial International EuroConference Biosignal 2000 in Brno, Czech Republic, 2000.

[10] T. Viero, K. Ostaimo, and Y. Neuvo, "Three-dimensional median-related filters for color image sequence filtering," IEEE Trans. on Circuits and Systems for Video Technology, vol. 4, no. 2, pp. 129-142, 1994.

[11] L. Jaroslavskij and I. Bajla, Methods and Systems of Digital Image Processing, Editorship of Technical and Economy Literature. ALFA, Bratislava, 1989.

[12] R. Lukáč, "An adaptive control of LUM smoother," Radioengineering, vol. 9, no. 1, pp. 9-12, 2000.

[13] N. R. Harvey and S. Marshall, "Optimum genetic algorithms for the design of stack filters," IEEE Trans. on Signal Processing, vol. 42, no. 4, pp. 832-835, 1994.

[14] R. Lukáč and S. Marchevský, "Adaptive LUM smoother controlled by adaptive threshold system," J. of Electr. Engrg., vol. 51, no. 3-4, pp. 100-104, 2000.

[15] R. Lukáč, New structures of LUM smoothers and impulse detectors for noisy images, Ph.D. thesis, Technical University of Košice, Slovak, 2001.
[16] R. Lukáč and S. Marchevský, "Adaptive vector LUM smoother," submitted to Proceedings of the IEEE Signal Processing Society ICIP 2001 in Thessaloniki, Greece, October 7-10, 2001, 2001.

[17] R. Lukáč, "Vector LUM smoothers as impulse detector for color images," submitted to Proceedings of European Conference on Circuit Theory and Design ECCTD '01 "Circuit Paradigm in the 21st Century" in Espoo, Finland, August 28-31, 2001, 2001.

[18] I. Pitas and A. N. Venetsanopoulos, "Order statistics in digital image processing," Proc. IEEE, vol. 80, no. 12, pp. 1892-1919, 1992.

[19] F. Palmieri and C. G. Boncelet, "Ll-filters-a new class of order statistic filters," IEEE Trans. on Acoustics, Speech, and Signal Processing, vol. 37, no. 5, pp. 691-701, 1989.

Rastislav Lukáč received the M.Sc. (Ing.) degree at the Technical University of Košice, the Slovak Republic, at the Department of Electronics and Multimedia Communications in 1998. In 2001 he finished Ph.D. study. Currently, he is assistant professor at the Department of Electronics and Multimedia Communications at the Technical University of Košice. His research interest includes image filtering, impulse detection, neural networks, and permutations.

Stanislav Marchevský received the M.Sc. degree in electrical engineering at the Faculty of Electrical Engineering, Czech Technical University in Prague, in 1976 and Ph.D. degree in radioelectronics at the Technical University of Košice in 1985. From 2001 he is the full professor at the FEI TU in Košice. His research interest includes image filtering, neural networks, genetic algorithms, and multimedia communication.
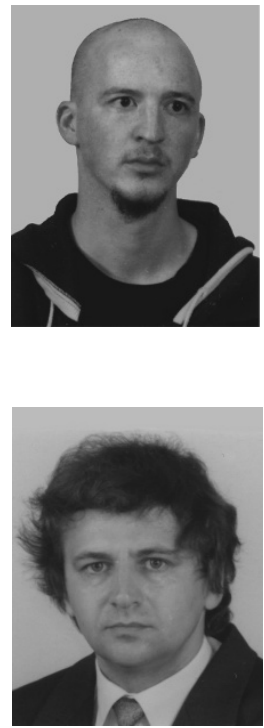